\title{
La Igualdad en el Lazo Social: Procesos Sociohistóricos y Nuevas Percepciones de la Desigualdad en la Sociedad Chilena
}

\author{
Kathya Araujo \\ Professora da Universidad Academia de Humanismo Cristiano no Chile. E-mail: \\ kathya.araujo@gmail.com
}

\section{INTRODUCCIÓN}

T a percepción social de las desigualdades requiere ser entendida en L referencia a los principios normativos de igualdad presentes en una sociedad. Para sustentar esta afirmación, basta recordar el hecho de que, como el debate lo ha subrayado repetidamente, no sería posible concebir las desigualdades tal como hoy las concebimos - el tipo de desigualdades que somos capaces de identificar o la falta de legitimidad que les atribuimos - si la igualdad no se hubiera instalado como fundamento de las expectativas en las llamadas sociedades modernas democráticas (Therborn, 2006) ${ }^{1}$; si la igualdad no se hubiera constituido en un componente esencial de la idea de justicia. A pesar de la casi evidencia de esta afirmación, en las investigaciones y debates en ciencias sociales, con frecuencia, esta interrelación no es estudiada de manera explícita ni teórica ni, menos aún, empírica (Reis, 2006; Turner, 1986). Aunque existe una masa muy significativa de estudios sobre desigualdades, no es este el caso de la igualdad.

Pero, todavía más, cuando la interrelación entre ambas es evocada, la mayoría de las veces esto ocurre a partir de un sesgo que podríamos llamar normativista. Las mayores contribuciones al debate sobre igualdad provienen del debate filosófico, ya sea en el marco de las dis-

DADOS - Revista de Ciências Sociais, Rio de Janeiro, vol. 56, no-1, 2013, pp. 109 a 132. 
cusiones sobre justicia en sus diferentes tradiciones liberales (Rawls o Dworkin), comunitaristas (Walzer) o desde la teoría crítica (Honneth). Consecuentemente, en buena parte de los estudios sociales, la igualdad elaborada filosóficamente funciona como premisa para la comprensión de las desigualdades. Como efecto de lo anterior, las experiencias sociales son contrastadas con un principio normativo abstracto despojado de contexto. El papel de los procesos sociales en la consolidación o especificación de la igualdad queda, de este modo, oscurecido. La paradoja, aquí, es, por cierto, que este abandono se ha dado a pesar de la evidencia histórica más que reiterada de que el principio de igualdad - y su función en la modernidad - no solo es un motor de las transformaciones sociales (Wagner, 1997), sino que es producto de un proceso histórico y social complejo (Rosanvallon, 2011; Dumont, 1983). En resumen, las desigualdades, al haber sido pensadas como fenómenos sociales complejos y dinámicos, han recibido profusa atención, mientras que la igualdad al haber sido mantenida como una construcción normativa abstracta ha tendido a ser abandonada a la filosofía. Lo que se oblitera en este movimiento es que no solo la percepción de las desigualdades es un problema sociológico, sino que lo es también el principio de igualdad. Es precisamente en este punto en el que se sitúa este texto.

La tesis que quiere defender este artículo, a partir de evidencia empírica, es que los contenidos de la igualdad, los que influyen en la percepción de las desigualdades, se especifican y transforman según los condicionantes estructurales e históricos de una sociedad. Para argumentar lo señalado, nos apoyaremos en resultados de investigaciones empíricas realizadas en Chile en los últimos años ${ }^{2}$. Nos proponemos discutir que el encuentro entre principios de igualdad que se difunden en el marco de su nueva ola expansiva en las últimas décadas y los rasgos propios a la sociedad chilena y sus determinaciones históricas configura al menos dos efectos relevantes e íntimamente vinculados. Por un lado, un proceso de especificación de la igualdad que se expresa en un conjunto de demandas de igualdad de un nuevo cuño que, más allá de la igualdad jurídica, política o social, se centran en lo que podríamos denominar como igualdad interactiva o igualdad en el lazo social. Por el otro, y en consonancia con lo anterior, da lugar a una aguda percepción de un tipo particular de desigualdades: aquellas que se despliegan en las interacciones sociales mismas.

El argumento se desarrollará en tres pasos. Empezaremos por explicitar brevemente los fundamentos del estudio social de la igualdad so- 
bre los que este trabajo se sostiene. Una vez realizado lo anterior, nos detendremos a situar el contexto histórico y social reciente en el caso de Chile desde la perspectiva de la expansión de los principios igualitaristas. En tercer lugar, abordaremos las transformaciones de las expectativas y los principios de legitimidad en las relaciones que se observan en esta sociedad, y sus efectos para la percepción de las desigualdades. Finalmente, y en conclusión, se extraerán las consecuencias de lo que estos procesos dicen respecto a las formas en que se transforma el principio de igualdad.

\section{EL ESTUDIO DE LA IGUALDAD}

La noción de igualdad ha sido normalmente discutida en asociación con el igualitarismo político y, en esa perspectiva, concebida como un principio moderno asociado al desarrollo del Estado nacional y de la idea de democracia y ciudadanía. En este contexto histórico, la idea de igualdad moderna, resultado de procesos sociales que se encarnan en luchas políticas (Rosanvallon, 2011:27-106), se instala como principio normativo. Esto quiere decir que funciona como una orientación de lo que la sociedad debería alcanzar y como medida para sus desempeños. La igualdad en tanto constituida como principio normativo de nuestra época no requiere de una justificación moral, como lo ha subrayado Berlin, aunque es necesario recordar que es un ideal entre otros (Wollheim y Berlin, 1956). Ella funciona proveyendo criterios para la evaluación de los trayectos que toma una sociedad al mismo tiempo que empuja a la generación de instituciones que resguarden la consonancia con el principio normativo y sus orientaciones (Turner, 1986).

Pero, si el principio normativo procede del dominio político, la igualdad es indisociable de procesos sociales - como bien lo intuyó y analizó Tocqueville cuando sostuvo que los caminos insospechados de la igualdad en la sociedad estadounidense conducían, más allá de toda voluntad política, a la creciente constitución de individuos (y masas) afectados por la "pasión" por la igualdad (Tocqueville, 1961). Recordar lo anterior subraya que existe un cierto nivel de autonomía y lógicas distintivas entre la función de la igualdad en el orden político y la función de la igualdad en las formas de organización de las relaciones e interacciones en una sociedad. A este nivel, sus contenidos, pero también los efectos de la igualdad, varían sustancialmente según los contextos. Estas variaciones se expresan en las formas particulares de percepción y enjuiciamiento de la realidad social que tienen los indivi- 
duos de una sociedad (por ejemplo en la percepción o no de un fenómeno como desigualdad), o en el régimen de lo admitido/prohibido que pone el marco a las actuaciones institucionales concretas. Lo anterior explica, por ejemplo, que mientras en algunas sociedades puede ser muy alto el rechazo a la exhibición de signos exteriores de riqueza, en otras, ello no solamente puede ser fuertemente tolerado sino incluso emulado; al mismo tiempo que, mientras en las primeras pueda haber modalidades de integración fuertemente jerarquizadas de grupos migrantes, las segundas revelen procesos de recepción más igualitaristas.

A esta primera distinción analítica - dominio político y lazo social - todavía es preciso añadirle una segunda: la diferencia entre principio normativo e ideal social. La igualdad es simultáneamente un principio normativo y un ideal social. El ideal social es una representación ofrecida a los individuos de lo deseable y valorado por una sociedad, y, por tanto, supone una definición atributiva sobre la manera en que el mundo y las personas deberían ser. El ideal es la contracara imaginaria de la dimensión simbólica que encarna el principio normativo. Así, el ideal social de igualdad llena de contenidos representacionales el significante igualdad que actúa como principio. Estos contenidos representacionales no son fijos sino que se van transformando. No solo en el largo plazo histórico, sino también - como lo muestran, por ejemplo, los resultados de una investigación empírica respecto a la noción de derecho - en el encuentro con las experiencias sociales que son propias a una sociedad e, incluso, al lugar social ocupado dentro de ella (Araujo, 2009).

Las dos caras de la igualdad pueden difícilmente ser pensadas la una sin la otra, por cierto, pero no se confunden. No todo ideal social es un principio normativo. Existen muchos ideales sociales que no consiguen convertirse en verdaderos ideales normativos, y menos aún son los que alcanzan a ser principios normativos en una sociedad o una cultura. Por ejemplo, si el derecho se ha constituido en nuestra época en un verdadero ideal normativo (Habermas, 1998), no podríamos decir lo mismo del ideal del ocio o de la vida sana. Pero, por el contrario, es imposible pensar en principios normativos que no estén sostenidos en la función del ideal. No solo porque la historia moderna muestra, como ya lo discutimos, que los principios normativos surgen de la movilización política de ciertos ideales, sino también porque, retomando las ideas de Weber en su discusión sobre autoridad (1964), es indispensable que se sostengan en la creencia - en la adhesión cognitiva-afectiva-de los individuos, pues ella es el fundamento de su legitimidad. Es 
desde este enfoque como abordaremos, en lo que sigue, y en el marco de la sociedad chilena, la cuestión de la igualdad.

\section{EXPANSIÓN DE LOS PRINCIPIOS IGUALITARISTAS Y PROCESOS SOCIOHISTÓRICOS LOCALES}

En Chile, como en otros países de América Latina, la igualdad como principio e ideal social ha sufrido una nueva ola expansiva en las últimas décadas. Lo anterior no quiere decir, de ningún modo, que los principios de igualdad como ideales no hayan tenido un proceso de expansión en la región de larga data - como lo atestiguan los movimientos sociales de comienzos del siglo XX (Salazar y Pinto, 1999) -, sino que este proceso adquiere nuevos contornos en la coyuntura histórica que se establece en las últimas tres a cuatro décadas, en el contexto de los procesos de democratización densamente discutidos por el debate académico (Dagnino, Olvera y Panfichi, 2006; Méndez, O'Donnell y Pinheiro, 2002, PNUD, 2004, entre otros). Tal como lo ha planteado Domingues (2009), si bien el ideario moderno de libertad igualitaria ha estado presente en América Latina como horizonte aproximadamente desde el mismo período en el que se instaló en Europa y en los Estados Unidos, los específicos procesos de la región permitirían hablar de una tercera fase de la modernidad que se desenvuelve en este último período en el que, a pesar de la hostilidad proveniente de la hegemonía del neoliberalismo, la ciudadanía se afirma mediante la movilización política y el sistema de derechos gana espacio en el imaginario y avanza en términos de institucionalización. Ahora bien, las fuentes de esta nueva ola expansiva del ideal de igualdad, como oferta a los sujetos para pensarse a sí mismos, se especifican en función de procesos sociales e históricos en el caso de Chile. Veámoslo con detalles.

Según lo muestran los resultados de la investigación realizada por Araujo y Martuccelli (2012), los individuos en Chile reconocen que las características más resaltantes del cambio histórico en el pasado reciente son, por un lado, la temprana instalación del modelo económico neoliberal y, por el otro, una renovada afirmación del horizonte de la democracia no solamente en términos políticos o institucionales, sino sobre todo a nivel de nuevas exigencias en el lazo social. El primer cambio los ha enfrentado a reformular su condición de sujetos económicos y laborales y a la ampliación de esferas que se desarrollan bajo la lógica de la mercantilización. El segundo ha impulsado una reconfiguración 
de los contenidos de la democracia y una comprensión particular de los procesos de democratización.

Respecto a lo primero, la sociedad chilena, con una intensidad inusitada desde 1973, fue el teatro exacerbado de uno de los grandes conflictos de las sociedades modernas: aquella que se da - retomando la caracterización de Karl Polanyi (1994) - entre la lógica del mercado y la lógica de la protección. A raíz del golpe de Estado de 1973, la sociedad chilena vivió una experiencia que hizo del país primero un "laboratorio" y luego un "modelo", uno de los primeros, del llamado orden neoliberal. Transitó, así, de un modelo centrado en el Estado a uno basado en el mercado, lo que implicó privatizaciones, liberalización económica, desregulación, subsidiariedad del Estado, apertura a la competencia internacional, flexibilidad laboral, entre otros.

La instauración del modelo no fue, sin embargo, lineal. El modelo conoció importantes correctivos. Tras la crisis de 1984 y la fuerte caída del Producto Interior Bruto en 1982 (Huneeus, 2000), se le dio más presencia a la acción estatal (Collier y Sater, 1999), y se orientaron las acciones de manera que los empresarios se constituyeron en un verdadero actor social (Montero, 1997). A esta primera inflexión, a partir de 1990, y con la vuelta de la democracia, se añadió una segunda. En ésta, los compromisos institucionales del Estado por medio de políticas sociales y ciertos esfuerzos, aunque limitados, de regulación se incorporaron. Para muchos analistas, es en este momento en que se consolidó el "milagro chileno".

En cualquier caso, el modelo produjo un rediseño de las fronteras entre el mercado y el Estado. Las relaciones entre los grupos sociales sufrieron una profunda transformación. La economía nacional se orientó a la exportación, que se abrió - de manera estratégica - al mercado internacional, y, más tarde, al mercado de capitales. El mercado de trabajo se reguló sobre nuevas bases, lo que permitió la creciente flexibilización y el ingreso de lógicas de competencia, a la vez que ocasionó el quiebre de la asociatividad colectiva en aras de una individualización creciente de las relaciones entre empleadores y empleados (Ramos, 2009; Soto, 2008; Todaro y Yañez, 2004). Se transformaron los principios de protección social y, más allá de ello, debido a la expandida privatización de la educación, la previsión social y la salud, se restringió el mapa de los servicios públicos a los cuales los ciudadanos pueden tener acceso. El consumo se convirtió en un fundamento estructural y de la defini- 
ción de status (Moulian, 1998) y el crédito en un elemento estructurador de las relaciones sociales y de la vida personal. Esto es, la transformación del capitalismo chileno supuso más el advenimiento de una nueva matriz que la simple "reducción" del Estado. En el corazón de estos procesos, lo que se produce es, más bien, como ha sido discutido, un nuevo modo de despliegue de las relaciones sociales (Hutton, 2003; Lordon, 2003; Berger, 2006).

Se instala, en consecuencia, la imagen de una sociedad perfectamente móvil y competitiva, la valorización de la ambición personal, de la confianza en el esfuerzo propio, la importancia del "empuje", de las "ganas" de tener éxito, una sociedad caracterizada por la oferta de integración por medio del consumo y que tiene al crédito como fundamento estructural de esta oferta. Por intermediación de las transformaciones promovidas por el modelo económico, como lo muestran nuestros resultados, se extiende en la sociedad una exigencia de hiperactuación para cada individuo ${ }^{3}$, pero, al mismo tiempo, se extiende, mediante la promesa del mérito, la filosofía de la competencia y la introducción activa del consumo y el crédito, una promesa igualitaria sui géneris. El mercado en Chile, bajo otras coordenadas que las que han sido discutidas para el caso europeo del siglo XVIII y XIX, se constituye en un gran factor de expansión en este momento histórico de la noción de igualdad situada en el individuo, la que se caracteriza más precisamente como igualdad de oportunidades en sus dos visiones: probabilista (por acción del azar) y posibilista (por acción del mérito) (Rosanvallon, 2011:315).

Veamos ahora la cuestión de la democratización. El colapso de la democracia a inicios de los setenta, y la instauración de una nueva matriz sociopolítica, así como el retorno a la democracia en los noventa participaron activamente en la reconfiguración y afirmación del horizonte democrático. La "democracia" en dictadura se va convirtiendo gradualmente para una parte no menor de la población en una esperanza y un objetivo que tienen como su exponente más relevante las luchas por el retorno a regímenes políticos democráticos (Vicuña et alii, 2001). La democracia se cargó, en este proceso, de legitimidad y urgencia. En el retorno a la democracia que se inicia con los gobiernos de la coalición Concertación por la Democracia, la "democracia", en cambio, es una tarea. Una tarea que tuvo límites, como se ha discutido. El primero es que ésta fue entendida principalmente en términos institucionales (Toloza y Lahera, 1998): modernización del Estado, transformación del 
sistema político y reconfiguración de las relaciones entre Estado y ciudadanía. El segundo de estos límites es que incluso en estos objetivos el alcance fue recortado, especialmente respecto al último. En este caso, y tal como lo han señalado diversos autores, no se implementaron sustentables y efectivos procesos de participación (De la Maza, 2002). Al contrario, se generaron estrategias de reducción del espacio público y se desarrolló una suerte de elitización y clausura creciente de la interlocución política (Garretón, 2000; Garretón y Garretón, 2010; JocelynHolt, 1999). No obstante, y esto es central, en función de contextos internacionales, factores políticos e históricos nacionales a nivel normativo, el discurso de la ciudadanía y la oferta de igualdad de derechos que le subtiende, en un contexto en que se privilegió la noción de equidad y de igualdad de oportunidades ${ }^{4}$, fueron fuertemente movilizados por el Estado.

La igualdad (en el marco de la expansión del ideal de derecho y la noción de ciudadanía) fue ofrecida normativamente como un valor principal para la sociedad y fue políticamente movilizada por el Estado (aunque, como ya se dijo, escasamente contemplada en la reformulación de las relaciones entre Estado y sociedad) y por el sistema político institucional (gradualmente también por los sectores conservadores, con fuerza en su versión de igualdad de oportunidades). Todo ello en un contexto de alta receptividad a las agendas de los organismos internacionales, al sistema de Naciones Unidas, principalmente, y otras instituciones, como el Banco Interamericano de Desarrollo y el Banco Mundial, con todas las enormes diferencias que puedan revelarse entre éstas. Así, se busca en los organismos internacionales una garantía de la democratización, legitimación internacional, y el acceso a recursos que se encuentran condicionados a estas agendas (Araujo, 2009b; Guzmán, 2002).

Pero, si la igualdad y la lucha por ella, aquí como en otros países de la región, ha estado fuertemente vinculada a la democracia (CEPAL, 2010), la dimensión político-institucional no alcanza para dar cuenta acabada de sus destinos. Las modalidades que ella toma en un contexto de democratización tienen que ser explicadas, también, y principalmente, por fuera del sistema político.

La expansión de la igualdad como ideal social tiene, en efecto, que ser entendida de manera particular a partir de factores sociales y culturales ${ }^{5}$. Mencionaremos aquí solo dos de ellos, de amplia, aunque no ex- 
clusiva, importancia. En primer lugar, ella ha sido impulsada por los movimientos sociales en Chile, los que juegan una función destacada que entronca precisamente con la lucha contra la dictadura y el retorno a la democracia y se especifican por el carácter transnacional de sus propias agendas y argumentos (Garretón, 2000). Por un lado, porque estos movimientos aportan a una ampliación de los campos a los que la igualdad como medida ha de ser aplicada sacándola de la pura dimensión socioeconómica para integrar otras dimensiones como el reconocimiento. Por el otro, porque ellos contribuyen en el marco de sus luchas a poner en el centro, y de manera renovada, la disputa por la cuestión de la ciudadanía, que, en última instancia, es la disputa, desde los inicios de la modernidad, por la igualdad. Un segundo aspecto es el aumento de la cobertura educacional. Este hecho no es menor porque, como ha sido discutido, la educación ha sido y continúa siendo un pilar de las ofertas de igualdad en Chile, bien visible en las aspiraciones de acceso a la educación superior (Espinoza, 2012). En síntesis, economía, política y transformaciones socioculturales aportan a la producción de un nuevo escenario de afirmación de la igualdad.

\section{EL TRABAJO DE LA IGUALDAD Y LA PERCEPCIÓN DE LAS DESIGUALDADES}

Los efectos de la expansión de los principios e ideales igualitarios en el contexto de los procesos descritos se verifican en nuestras investigaciones. Para que un ideal resulte eficiente debe estar inscrito a nivel de los individuos. Pues bien, como lo ha mostrado un estudio sobre las modalidades en que la noción de derecho era movilizada por los individuos para entender el mundo, orientar sus actos y legitimar sus acciones en la vida cotidiana, la igualdad es un ideal social presente en los ideales del "yo", pues constituye una herramienta de lectura y de evaluación de la justicia en la sociedad (Araujo, 2009a). Vale la pena subrayar que de lo que se trata aquí es que la igualdad no es ya solamente un principio normativo a disposición de algunos actores colectivos o de "vanguardias" iluminadas, sino que es una referencia presente y constante en el juicio y evaluación que hacen individuos ordinarios de las experiencias cotidianas que tienen en su sociedad.

Pero, el estudio antes referido mostró, al mismo tiempo, que el trabajo del ideal solo puede ser capturado en su articulación con las experiencias sociales. Los ideales se entraman con lo que los individuos "saben" (un saber no consciente necesariamente) de las lógicas actuantes 
en una sociedad. Se articulan con el saber sobre la vida social que decanta de las experiencias sociales de los individuos en una sociedad. $Y$ esto influye en la capacidad de acción del ideal, los efectos que tendrá, pero también, como veremos, en los contenidos específicos que este toma. A este respecto, los resultados mostraron que, en Chile, las experiencias sociales ponen permanentemente en cuestión el funcionamiento efectivo de la igualdad. Por cierto, esta tensión entre principio de igualdad y desigualdades no es propia de Chile: es constitutiva de las sociedades capitalistas modernas (Turner, 1986) y se podría incluso argüir de toda sociedad. Pero, y es aquí donde el estudio empírico de la igualdad ilumina diferencias, ella no se da del mismo modo en toda sociedad como tampoco tiene las mismas consecuencias.

Si, como lo ha discutido Rosanvallon (2011), la cuestión fundamental de esta tensión en sociedades europeas - como la francesa hoy - es la brecha que hay entre un sentimiento fuerte de rechazo a las desigualdades económicas y sociales al mismo tiempo que una aceptación tácita de los mecanismos que la producen y por tanto una escasa disposición a la acción correctiva, no es necesariamente el punto más álgido en una sociedad latinoamericana como la chilena. La tensión entre principio de igualdad y desigualdades ilumina en Chile, como veremos, las contradicciones entre una sociedad que se postula como moderna e igualitaria, que incluso propone y sostiene mecanismos institucionales orientados a la igualdad, pero que lo hace sobre la experiencia de un lazo social profundamente vertical y jerárquico y de unos individuos que imbuidos de las promesas de la igualdad buscan sin éxito su verificación precisamente a este nivel.

Los individuos en Chile identifican como sustrato de estas experiencias de negación de la igualdad, la actuación de cuatro lógicas sistémicas que ordenan las relaciones sociales:

1) La lógica de las jerarquías naturalizadas (Bengoa, 2006; ICSO, 2010), lo que supone la mantenida importancia de los rasgos adscritos y de una arquitectura relacional fuertemente vertical.

2) La lógica de los privilegios. Particularmente asociada a razones de clase, se expresa en experiencias que desmienten la meritocracia (Navia y Engel, 2006), en la permanente necesidad de nivelación por medio de recursos como el "pituto" (movilización informal de influencias) (Barozet, 2006) o en el hecho de que el apellido y las re- 
La Igualdad en el Lazo Social: Procesos Sociohistóricos y Nuevas Percepciones...

des familiares sean centrales para definir las oportunidades (Núñez y Gutiérrez, 2004).

3) La lógica de la confrontación de poderes. El espacio social es percibido como un espacio de enfrentamiento de poderes, en el cual el abuso es una constante debido a la desregulación de estas relaciones. Se expresa en la movilización constante aunque cauta de signos de poder, los juegos de "tasación" y las estrategias sociales de cálculo y evitación que gobiernan las relaciones (Araujo, 2009a).

4) La lógica del autoritarismo y la desestimación de la autoridad. El autoritarismo es una clave de comprensión pero también de acción extendida. El modelo de autoridad se confunde con el modelo del autoritarismo finalmente. La relación con las normas en este contexto aparece necesariamente como una imposición acatada pasivamente, y no como un consentimiento activo.

El privilegio legitima las diferencias basadas en las jerarquías consideradas naturales a las que se opone la igualdad desde una perspectiva histórica y sociológica (Dumont, 1983). El uso desregulado del poder, interfiere en el desarrollo de la confianza indispensable para la coordinación social, define ejercicios de poder que obstaculizan la gestión de las jerarquías (autoridad) y bloquea la posibilidad del establecimiento de un espacio común entre los individuos más allá de sus particularidades. En palabras de uno de nuestros entrevistados:

Esta sociedad todavía es muy segmentada, si bien es una sociedad más abierta que antes, sigue siendo muy jerárquica, muy poco permeable, hay poca movilidad social en general, o sea, la gente te identifica por la cara, por el color del pelo, te identifica por la ropa que vistes, entonces hay mucha tendencia a tipificar, a encasillar, mucho prejuicio (hombre, sectores medios).

Lo que resulta importante subrayar es que todas estas lógicas sistémicas están presentes en la experiencia social cotidiana, desde la experiencia en el transporte público hasta las interacciones y funcionamiento de la política pasando por las interacciones en los servicios de salud públicos o las escuelas. Por tanto, todas estas experiencias ponen en cuestión de manera encarnada y continuada el principio de igualdad.

¿Qué es lo que ha resultado de este encuentro entre la expansión de la igualdad y su creciente inscripción en los individuos y las lógicas sistémicas que dan cuenta de la vigencia de ciertas formas de estructura- 
ción jerárquica y verticalizante de la sociedad (Bengoa, 2006; Larraín, 2001; Salazar y Pinto, 1999)? Como lo revela una nueva y reciente investigación (Araujo y Martuccelli, 2012), este encuentro ha resultado en una traducción particular de los principios igualitaristas en la vida social: la aparición de acuciantes expectativas de horizontalidad, las que, a su vez, impactan directamente en las formas de percepción de las desigualdades.

\section{Las Expectativas de Horizontalidad ${ }^{6}$}

La expansión de los principios de igualdad es de larga data, es evidente. También lo es, y está aún vigente, la primera manera en que dicha expansión se ha encarnado en nuestras sociedades, esto es, el modo de su traducción en grandes principios políticos o jurídicos, asociada a demandas de inclusión política, horizonte de los movimientos sociales ${ }^{7}$. Pero, hay una segunda manera en que la igualdad se encarna en la actualidad en Chile y es la que los resultados de nuestra investigación muestran como novedad. La igualdad se traduce, hoy, en un reclamo ampliamente generalizado de horizontalidad en el lazo social. No nos referimos con ello, por cierto, a la expectativa de un mundo horizontal, sino a la de un trato horizontal tanto en relaciones simétricas como asimétricas. Es la horizontalidad en los intercambios cara a cara la que se convierte en una exigencia central. La horizontalidad es un anhelo interactivo y se especifica en la búsqueda de una sociabilidad más horizontal. Ello implica la demanda de una gestión de las asimetrías con un fundamento indiscutible horizontal, pero, también, que desaparezca la tendencia a la asimetrización verticalizante (Güell, 2012) de las relaciones por medio de privilegios o uso de recursos del poder. Se trata de una exigencia dirigida tanto a las instituciones como a los otros.

Se trata, entonces, de una revolución de las expectativas, que toca lo más común porque involucra la transformación misma del lazo social, pero atañe también a lo más íntimo porque compromete los signos de la dignidad personal y es hoy por hoy un índice privilegiado del respeto. A partir de ella se exige una modificación generalizada del trato estatutario entre todos los individuos. El cumplimiento de esta expectativa intervendrá de manera decidida en perfilar los juicios acerca de las instituciones y de las personas. Es en este sentido que la horizontalidad que irrumpe en la sociedad chilena se expresa a nivel de la eclosión de una nueva sensibilidad, una que se vincula con la percepción agu- 
zada de las desigualdades que se despliegan en las interacciones sociales.

\section{Desigualdades en las Interacciones Sociales}

Nuestros estudios muestran que, en el caso de Chile, la percepción de las desigualdades se encuentran fuertemente asociadas a una extendida denuncia de la verticalidad, la jerarquía y su abuso. Ello se basa en su experiencia frente a situaciones tan primarias como no ser tratados con cortesía o ser apelados peyorativamente como "huevones flojos" por sus superiores en el mundo laboral, por ejemplo. Dicho por una mujer quien relata sus experiencias en su lugar de trabajo: "Es un maltrato que es con... es maltrato que va desde un tuteo pesado [...] al decirte 'anda a sacar fotocopias'; y te das cuenta que a la otra persona le dicen 'vaya a sacar fotocopias', y a ti 'anda'" (mujer adulta, sectores populares).

Aunque las experiencias de abuso no son homogéneas ni se reparten por igual en todos los ámbitos de la vida cotidiana, de manera transversal, el abuso es denunciado, rechazado, sufrido en carne propia de manera masiva. Lo central aquí es la conciencia aguzada del abuso en las relaciones sociales. Conciencia aguzada quiere decir la presencia de un alerta y aún sobre-alerta en la relación con los otros. Esta alerta es el fundamento de la denuncia, cierto, pero también, en su faz de sobre-alerta es el combustible para la reacción irritada y aún abusiva respecto del otro (la presuposición de estar siendo abusado o de la predisposición del otro a abusar de uno funciona como premisa que anima y justifica el propio acto abusivo). El abuso es el omnipresente fantasma social. El lenguaje del abuso y de la falta de respeto se ha convertido en expresión natural para designar lo que se percibe, cada vez más, como el fruto de actitudes social y moralmente intolerables. Como dice un hombre joven: "Yo siento que se aprovechan de eso [de su poder]. Yo entiendo que ahí está el problema, que uno no tiene cómo defenderse, entonces son como un bicharraco que te puede atacar y tú no tienes ninguna decisión que ponerle" (hombre, sectores medios).

¿Lo que subyace a todas estas experiencias? El conflicto con el poder y la verticalidad. En todo orden igualitario es preciso saber lidiar con relaciones verticales, como lo ha mostrado Goffman, pero en Chile esto tiene sus particularidades. Estos abusos y estos maltratos son concebidos como resultados de remanencias de un orden jerárquico y una no- 
ción de verticalidad sostenida en la suposición de superioridad naturalizada de quienes ocuparían el vértice superior en la escala social, rasgos del patrón de interacción social históricamente producido y culturalmente reproducido.

Ahora bien, y esto es extremadamente importante, si bien estas remanencias son percibidas como actuantes, también han perdido legitimidad. Esta pérdida de legitimidad tiene dos grandes efectos. Por un lado, como lo indican los resultados de una investigación reciente ${ }^{8}$, aportan a una generalización de la desconfianza respecto de ella. La autoridad, en todas sus formas, deja de ser vista desde la perspectiva de la regulación del ejercicio del poder por intermedio de la legitimidad (Weber, 1964) o como el plus que sanciona y pacifica el sometimiento al poder (Renaut, 2004). Pasa a ser percibida desde otra perspectiva, notablemente desde su exceso o su arbitrariedad, esto es, en clave de autoritarismo: "Chile es así como un país bien autoritario, bien autoritario. Pero...no así al 100\%, un 70\%, porque el otro como que no pesa mucho..." (mujer, sectores populares).

De manera reveladora, en la perspectiva de esta mujer, ese treinta por ciento no es el que ejerce la autoridad en un modo no autoritario sino que refiere a aquellos que "no están ni allí" con la autoridad. El reclamo de horizontalidad, y su insistencia por un trato horizontal en la simetría y la asimetría, exige producir bajo nuevas bases los fundamentos de la autoridad tanto en el ámbito político como familiar o laboral. Pone bajo tensión uno de los pilares regulatorios de las relaciones de poder en la sociedad. Por otro lado, dada la pérdida de legitimidad de las lógicas sistémicas que les subyacen, estas experiencias se constituyen en signos privilegiados de la percepción de la injusticia en la sociedad, lo que explica la sensibilidad aguzada a este tipo de desigualdades.

De este modo, la revolución democratizante en curso en la sociedad chilena, cuyo contenido principal son las expectativas de horizontalidad en el lazo social en su encuentro con lógicas sistémicas que las contradicen, permite explicar que las denuncias de los abusos ejercidos en el trato diario o los maltratos en los encuentros cara a cara sean contenidos relevantes en la percepción de la desigualdad. Llamaremos a este tipo de desigualdad interaccional ${ }^{9}$.

Las desigualdades interaccionales son desigualdades que se expresan y perciben sobre todo a nivel del lazo social en las interacciones coti- 
dianas y corrientes entre individuos y entre éstos y las instituciones. La percepción de la desigualdad interaccional no desplaza en importancia a las percepciones de desigualdad económica y jurídica, pero se convierte en el barómetro principal desde el cual, particularmente en los sectores populares, las otras desigualdades son leídas ${ }^{10}$.

Por supuesto, vale aquí hacer la pregunta, ¿por qué no usar para definir este fenómeno, por ejemplo, el término de desigualdades existenciales acuñado por Therborn ${ }^{11} \mathrm{o}$ asociarlo a las teorizaciones sobre reconocimiento de Honneth en vez de optar por el de desigualdades interaccionales? Sin duda, las desigualdades interaccionales implican o se relacionan con cada una de aquellas dimensiones. No obstante, no pueden ser absorbidas por ellas. ¿Por qué? Por tres razones.

La primera: porque el reconocimiento - y a diferencia de aquellos que lo han trabajado desde el problema de la justicia y poniendo el acento en la identidad o el status respectivamente (Honneth, 1997; Taylor, 2003; Fraser en Fraser y Honneth, 2006) - más que ser un elemento central, en el caso estudiado, es solo uno de los varios elementos que constituyen la gramática de lo que sustenta el "buen trato" exigido en la interacción social.

La segunda: porque las desigualdades que situamos en este trabajo están lejos de constituirse o aún de dejarse articular por lo jurídico y lo institucional, lo que sí es el caso de muchas de las desigualdades producidas en torno al reconocimiento. Este tipo de desigualdades, las que el caso de Chile permite situar, están focalizadas en los patrones de interacción social. Es decir, atañen al orden de la sociabilidad y la civilidad. Vale la pena insistir: caen fuera de los regímenes de tratamiento jurídico e institucional. Esto resulta decisivo.

Una tercera razón para insistir en la noción de desigualdades interaccionales, y no subsumirla en una definición más general como desigualdades existenciales, es que ella permite iluminar, y sacar las consecuencias de uno de los rasgos específicos de las sociedades latinoamericanas: el peso que tiene en ellas la sociabilidad. Es esta una importancia que ha sido resaltada desde diferentes canteras del pensamiento latinoamericano, desde los llamados ensayos del carácter (Paz, 1987) pasando por los textos fundamentales del pensamiento social (Freyre, 2010 o Buarque de Holanda, 2006), hasta llegar a interpretaciones más contemporáneas (Nugent, 1992; Nino, 2005; Adler-Lomnitz, 1971, entre otros). En todos ellos y en otros más, la sociabilidad ha sido destaca- 
da como central para entender la dimensión de integración social (Lechner, 1987; Morandé, 1984) en la región, al mismo tiempo que como escenario privilegiado de los juegos dirimentes de poder social, como lo mostró también, por ejemplo, el famoso debate entre Roberto Da Matta (2002) y Guillermo O'Donnell (1984). Dada esta centralidad, la dimensión de la interacción social se constituye en un espacio destacado en el que se juega y ha jugado en nuestra región lo que Rancière (2007a) ha llamado la "verificación de la igualdad". La noción de desigualdades interactivas, y el proceso a que ella hace referencia, pone el foco en uno de los destinos posibles de una tensión largamente diagnosticada en la región: la disonancia entre procesos modernizadores económicos y culturales y el funcionamiento de relaciones sociales de carácter jerárquico y vertical que se han mantenido por mucho tiempo intocadas.

\section{CONCLUSIONES}

Los procesos socio-históricos en su encuentro con los principios normativos no solo van a participar en definir las coordenadas de la percepción de lo que es concebido como desigualdades, así como la jerarquía en la percepción de las mismas, sino que, simultáneamente, aportan a la definición de los contenidos específicos que toma el principio de igualdad en su actuación en la sociedad.

Como vimos, en Chile, la igualdad aparece como un elemento muy relevante a partir del cual es pensada y evaluada la sociedad y a uno mismo en ella. Pero ella, además, se aplica a un nuevo ámbito. El funcionamiento de la igualdad en una sociedad se mide calculando el grado en que el principio normativo de la igualdad ha permeado la forma de distribución de recursos (Ottone y Vergara, 2007) o en que se me concibe en comparación con otros ante la ley (Méndez, O'Donnell y Pinheiro, 2002). En esto Chile no es una excepción, pero - y esto es lo que muestran nuestros resultados -, también se mide, y de manera aguzada, por la manera en que ha conseguido o no permear el patrón de las interacciones sociales: el trato que recibido por el otro, las formas de ejercicio de la autoridad, las condiciones para el reconocimiento, el grado de amabilidad o desconsideración.

Las exigencias, entonces, se concentran en un nuevo ámbito. Éstas son convocadas de manera espontánea por las personas, menos en su sentido abstracto jurídico o político o desde el nivel estructural económico 
(aunque lo segundo está mucho más presente que lo primero), y mucho más desde una perspectiva fuertemente vinculada con las experiencias sociales en las interacciones sociales. Esto es, estamos ante una noción de igualdad en acción que aunque apoyándose en ellas trasciende a la igualdad jurídica y la igualdad social. Esta nueva figura del principio de igualdad desborda lo producido por los discursos institucionales y políticos. Se trata de la igualdad aplicada a la dimensión interactiva del lazo social, o la igualdad en el lazo social.

Pero, los resultados no solo revelan que la igualdad se asocia a un nuevo ámbito, sino que también se definen las formas de medición de su actuación de manera novedosa. Todos somos iguales en la medida en que recibimos el mismo trato en las interacciones cotidianas independientemente de la posición social, los signos de distinción que podamos movilizar o la relación al poder que podamos ostentar. De esta manera, el índice particular para la medición de la igualdad en el lazo social es la horizontalidad en las relaciones sociales. Las formas de la sociabilidad y la civilidad se convierten en un barómetro privilegiado de las injusticias ${ }^{12}$. La igualdad en el lazo social y la exigencia de horizontalidad que le es consustancial impulsa un nuevo ámbito de demanda y de exigencia ciudadana, y hoy por hoy, se constituye en un aspecto candente de la democracia en Chile.

(Recebido para publicação em outubro de 2012)

(Reapresentado em dezembro de 2012)

(Aprovado para publicação em janeiro de 2013) 


\section{NOTAS}

1. Para el caso europeo, la desigualdad como una forma mayor de injusticia aparece solo en el contexto de la modernidad en la que los seres humanos son concebidos a partir de la idea de un sustrato común y de igualdad de base (Therborn, 2011).

2. La primera: Noción de derechos en los sectores medios y de menores recursos (2003-2007). Estudio de las modalidades en que la noción de derecho e igualdad es movilizada en la percepción, evaluación y actuación en lo social a partir de la realización de 22 Grupos de Conversación Dramatización, cada cual de entre 5 a 8 participantes, desarrollada en la Región Metropolitana. Se consideraron en la elaboración muestral criterios de sexo, generacionales (jóvenes y adultos) y socio-económicos (sectores medios y sectores populares). La segunda: Procesos de individuación y configuración de sujeto en la sociedad chilena actual. Proyecto FONDECYT no 1085006 (2007-2010). En este caso, se trata de un estudio sobre las determinaciones estructurales y procesos de individuación en curso en la sociedad chilena, basado en la realización de 96 entrevistas semi-directivas en tres ciudades chilenas, con hombres y mujeres de entre 30 y 55 años de sectores populares y sectores medios. La tercera: Autoridad y procesos de democratización social en Chile. Proyecto FONDECYT no 1110733 (a partir del 2011 y en curso). Este último estudio versa sobre las modalidades de ejercicio de la autoridad y razones para la obediencia en el contexto de los procesos de democratización social reciente, focalizado en el ámbito del trabajo y la familia. Para este artículo se hizo uso de los resultados del análisis de 32 entrevistas semi-directivas, las que fueron realizadas a hombres y mujeres de entre 30-55 años, de sectores populares y sectores medios.

3. Cf. Araujo y Martuccelli, 2012, tomo II, pp. 244-249.

4. Para una temprana discusión crítica de esta tensión entre igualdad y equidad y el uso de esta última por el Estado, ver Garretón (1999).

5. El estudio de los mecanismos y procesos de expansión sociocultural de la igualdad como ideal y/o como principio normativo es un tema que requiere aún de mayores estudios empíricos detallados en el caso de Chile.

6. Para una discusión detallada ver Araujo y Martuccelli (2012: 83-121).

7. Para el caso de Chile, Garcés (2004).

8. Autoridad y procesos de democratización social en Chile, Proyecto FONDECYT $\mathrm{n}$ 은 1110733 (ver nota 2).

9. Garretón y Cumsille (2002), encontraron en un estudio sobre percepción de desigualdades que los jóvenes de nivel socioeconómico bajo priorizaban la desigualdad como injusticia social y abuso de poder. Este mismo estudio mostró que, respecto a la percepción de las mayores desigualdades, el $87 \%$ de los encuestados señalaban que eran las relativas a personas con poder y sin poder. Estos resultados hacen que los autores mencionen la existencia de lo que denominan desigualdades ciudadanas o cívicas, sin embargo este concepto no es retomado de manera sistemática posteriormente. En rigor, la denominación ciudadana termina siendo restrictiva porque los fenómenos a los que estos resultados apuntan no pueden ser absorbidos completamente por la noción de ciudadanía ordenada en términos de deberes y derechos.

10. Es posible que estos resultados aporten a la explicación del hecho de que cuando se analizan las percepciones de desigualdad económica, la magnitud de la percepción 
La Igualdad en el Lazo Social: Procesos Sociohistóricos y Nuevas Percepciones...

de los sectores populares sea menor que la de otros (Castillo, 2009; Castillo, Miranda y Carrasco, 2011). Visto desde nuestros resultados, es posible suponer que se trataría menos de una dificultad perceptiva de estos sectores que de un efecto de la importancia que tienen estas desigualdades interaccionales en la jerarquía de las desigualdades percibidas.

11. Como se sabe, este autor, reverberando otros trabajos como el de Honneth (1997) o Margalit (1999), define este tipo de desigualdades como desigual reconocimiento de los seres humanos como personas, lo que tiene efectos en la distribución de libertad para continuar los proyectos y en la afirmación o negación del reconocimiento y respeto. Una desigualdad que puede ser reconocida como tal en la medida en que el sentimiento de humillación, de no ser reconocido y no ser respetado, esté presente (Therborn, 2006).

12. Un argumento adicional, que no nos es posible desarrollar aquí puede extraerse de la observación de los fenómenos políticos actuales, entre ellos, las retóricas de las nuevas demandas y movilizaciones sociales, las modalidades de construcción de los liderazgos sociales, o el esfuerzo muchas veces poco consciente de apropiación de estos lenguajes sensibles a la desigualdad interaccional de la clase política tradicional en su intento de ganar el favor electoral. 


\section{BIBLIOGRAFÍA}

ARAUJO, Kathya. (2009), Habitar lo Social. Usos y Abusos en la Vida Cotidiana en el Chile Actual. Santiago, LOM Ediciones.

(2009a), “Los Procesos de Transnacionalización como Procesos de Recepción. Agendas Transnacionales y Contextos Locales en el Caso de Chile". Universum. Revista de Humanidades y Ciencias Sociales, vol. 2, pp.13-28.

. (2009b), "Configuraciones de Sujeto y Orientaciones Normativas". Psicoperspectivas, vol. VIII, no 2, pp. 248-265.

ARAUJO, Kathya y MARTUCCELLI, Danilo. (2012), Desafíos Comunes. Retrato de la Sociedad Chilena y sus Individuos (2 tomos). Santiago, LOM Ediciones.

BAROZET, Emmanuelle. (2006), “El Valor Histórico del Pituto: Clase Media, Integración y Diferenciación Social en Chile". Revista de Sociología, no 20, pp. 69-96.

BENGOA, José. (2006), La Comunidad Reclamada. Identidades, Utopías y Memorias en la Sociedad Chilena Actual. Santiago, Catalonia.

BERGER, Suzane. (2006), Made in Monde. Paris, Seuil.

BUARQUE DE HOLANDA Sérgio. (2006), Raízes do Brasil. São Paulo, Companhia das Letras.

CASTILLO, Juan Carlos. (2009), “Cuál es la Brecha Salarial Justa? Opinión y Legitimidad de la Desigualdad Económica en Chile". Estudios Públicos, no 113, pp. 237-266.

CASTILLO, Juan Carlos, MIRANDA, Daniel y CARRASCO, Diego. (2011), La Percepción Desigual de la Desigualdad. Santiago, Pontificia Universidad Católica de Chile, Centro de Medición Mide.

CEPAL (COMISIÓN ECONÓMICA PARA AMÉRICA LATINA). (2010), Panorama Social de América latina. La Hora de la Igualdad: Brechas por Cerrar, Caminos por Abrir. Santiago de Chile, CEPAL.

COLLIER, Simon y SATER, William. (1999), Historia de Chile 1808-1994. Madrid, Cambridge University Press.

DAMATTA, Roberto. (2002), Carnavales, Malandros y Héroes. México, Fondo de la Cultura Económica.

DAGNINO Evelina, OLVERA Alberto J. y PANFICHI, Aldo. (2006), La Disputa por la Construcción Democrática en América Latina. México, FCE, CIESAS, Universidad Veracruzana.

DE LA MAZA, Gonzalo. (2002), “Los Movimientos Sociales y la Democratización en Chile", en P. Drake y I. Jaksic (orgs.), El Modelo Chileno. Democracia y Desarrollo en los Noventa. Santiago, LOM Ediciones, pp. 377-405.

DOMINGUES, José Maurício. (2009), La Modernidad Contemporánea en América Latina. Buenos Aires, Siglo Veintiuno.

DUMONT, Louis. (1983), Essais sur l'Individualisme. Paris, Seuil.

ESPINOZA, Vicente. (2012), "El Reclamo Chileno contra la Desigualdad de Ingresos". Revista Izquierdas, no 12, pp. 1-25. Disponible en: http:/ / www.izquierdas.cl. 
La Igualdad en el Lazo Social: Procesos Sociohistóricos y Nuevas Percepciones...

FRASER Nancy y HONNETH, Axel. (2006), ¿Redistribución o Reconocimiento? Madrid, Ediciones Morata.

FREYRE, Gilberto. (2010), Casa Grande e Senzala. Madrid, Marcial Pons.

GARCÉS, Mario. (2004) “Los Movimientos Sociales Populares en el Siglo XX: Balance y Perspectivas". Política, no 43, pp. 13-43.

GARRETÓN, Manuel Antonio. (1999), “Igualdad, Ciudadanía y Actores en las Políticas Sociales". Revista de Ciencias Sociales, no 9, pp. 41-52.

. (2000), La Sociedad en que Vivi(re)mos. Santiago, LOM Ediciones.

y CUMSILLE, Guillermo. (2002), "Las Percepciones de la Igualdad en Chile". Proposiciones, vol. 34, pp. 64-76.

GARRETÓN, Manuel Antonio y GARRETÓN Roberto. (2010), “La Democracia Incompleta en Chile: La Realidad tras los Rankings Internacionales". Revista de Ciencia Política, vol. 30, no 1, pp. 115-148.

GÜELL, Pedro. (2012), “La Producción de la Desigualdad: Orden, Discurso, Subjetividad", en V. Lezcano, W. Enedino y E. Nolasco (eds.), Estudos de Linguagens: Diversidade e Ensino. São Paulo, Pedro \& João Editores.

GUZMAN, Virginia. (2002), Las Relaciones de Género en un Mundo Global. Santiago de Chile, Documento CEPAL (Serie Mujer y Desarrollo).

HABERMAS, Jürgen. (1998), Facticidad y Validez. Madrid, Trotta.

HONNETH, Axel. (1997), La Lucha por el Reconocimiento. Barcelona, Crítica.

HUNEEUS, Carlos. (2000), El Régimen de Pinochet. Santiago, Editorial Sudamericana.

HUTTON, Will. (2003), The World We're In. London, Abacus.

ICSO (Instituto de Investigación y Ciencias Sociales). (2010), Chile 2009: Percepciones y Actitudes Sociales. Santiago, Universidad Diego Portales.

JOCELYN-HOLT, Alfredo. (1999), El Peso de la Noche. Santiago, Planeta.

LARRAIN, Jorge. (2001), Identidad Chilena. Santiago, LOM Ediciones.

LECHNER, Norbert (comp.). (1987), Cultura Política y Democratización. Buenos Aires, CLACSO.

LOMNITZ, Larissa Adler. (1971), “Reciprocity of Favors in the Urban Middle Class of Chile", en G. Dalton (comp.), Studies in Economic Anthropology. Washington, American Anthropological Association, pp. 93-106.

LORDON, Frédéric. (2003), La Politique du Capital. Paris, Odile Jacob.

MARGALIT, Avishai. (1999), La Sociedad Decente. Barcelona, Paidós.

MENDEZ, Juan, O’DONNELL Guillermo y PINHEIRO, Paulo (comps.). (2002), La (in)efectividad de la Ley y la Exclusión en América Latina. Buenos Aires, Paidós.

MONTERO, Cecilia. (1997), La Revolución Empresarial Chilena. Santiago, Dolmen Ediciones.

MORANDE, Pedro. (1984), Cultura y Modernización en América Latina. Santiago de Chile, Instituto de Sociología, Universidad Católica de Chile. 


\section{Kathya Araujo}

MOULIAN, Tomás. (1998), Chile Actual: Anatomía de un Mito. Santiago, LOM Ediciones.

NAVIA, Patricio y ENGEL, Eduardo. (2006), Que Gane el Más Mejor. Mérito y Competencia en el Chile de Hoy. Santiago, Debate.

NINO, Carlos. (2005), Un País al Margen de la Ley. Buenos Aires, Emecé.

NUGENT, Guillermo. (1992), El Laberinto de la Choledad. Lima, Fundación Friedrich Ebert.

NUÑEZ, Javier y GUTIERREZ, Roberto. (2004), “Class Discrimination and Meritocracy in the Labor Market: Evidence from Chile". Estudios de Economía, vol. 31, no 2, pp.113-132.

O'DONNELL, Guillermo. (1984), ¿Y a míquéme Importa? Notas sobre Sociabilidad y Política en Argentina y Brasil. Buenos Aires, CEDES.

OTTONE, Ernesto y VEGARA, Carlos. (2007), “La Desigualdad Social en América Latina y el Caso Chileno". Estudios Públicos, no 108, pp. 59-91.

PAZ, Octavio. (1987), El Laberinto de la Soledad. México, FCE.

PNUD (Programa de las Naciones Unidas para el Desarrollo). (2004), La Democracia en América Latina. Hacia una Democracia de Ciudadanas y Ciudadanos. Lima, PNUD.

POLANYI, Karl. (1994), The Great Transformation. Boston, Beacon Press.

RANCIÈRE, Jacques. (2007a), En los Bordes de lo Político. Buenos Aires, Editorial La Cebra.

(2007b), El Odio a la Democracia. Buenos Aires, Amorrortu.

RAMOS, Claudio. (2009), La Transformación de la Empresa Chilena. Una Modernización Desbalanceada. Santiago, Editorial Universidad Alberto Hurtado.

REIS, Elisa. (2006), "Inequality in Brazil: Facts and Perceptions", en G. Therborn (ed.), Inequalities of the World. London, Verso.

RENAUT, Alain. (2004), La Fin de l'Autorité. Paris, Flammarion.

ROSANVALLON, Pierre. (2011), La Société des Égaux. Paris, Seuil.

SALAZAR, Gabriel y PINTO, Julio. (1999), Historia Contemporánea de Chile. Volumen 2: Actores, Identidad y Movimiento. Santiago, LOM Ediciones.

SOTO, Álvaro. (2008), Flexibilidad Laboral y Subjetividades. Hacia una Comprensión Psicosocial del Empleo Contemporáneo. Santiago, LOM Ediciones.

TAYLOR, Charles. (2003), El Multiculturalismo y "La Política del Reconocimiento". México, Fondo de Cultura Económica de España.

THERBORN, Göran. (2011), “Inequalities and Latin America. From the Enlightenment to the 21st Century". Working Paper Series, no 1. Disponible en: http:/ / edocs.fu-berlin. de / docs / servlets / MCRFileNodeServlet/FUDOCS_derivate 000000001967/1_WP_Therborn_online.pdf?hosts=

. (2006), “Meaning, Mechanisms, Patterns, and Forces of Inequalities. An Introduction", en G. Therborn (ed.), Inequalities of the World. New Theoretical Frameworks. Multiple Empirical Approaches. London, Verso.

TOCQUEVILlE, Alexis de. (1961), De la Démocratie en Amérique. Paris, Gallimard, 2 vols. 
La Igualdad en el Lazo Social: Procesos Sociohistóricos y Nuevas Percepciones...

TODARO, Rosalba y YAÑEZ, Sonia (eds.). (2004), El Trabajo se Transforma. Relaciones de Producción y Relaciones de Género, Santiago, CEM.

TOLOZA, Cristián y LAHERA Eugenio. (1998), Chile en los Noventa. Santiago, Dolmen Ediciones.

TURNER, Bryan. (1986), Equality. Sussex/London, Ellis Horwood/Tavistock.

VICUÑA, Manuel et alii. (2001), Historia del Siglo XX Chileno. Santiago de Chile, Sudamericana.

WAGNER, Peter. (1997), Sociología de la Modernidad. Barcelona, Herder.

WEBER, Max. (1964), Economía y Sociedad. México, FCE.

WOLLHEIM, Richard y BERLIN, Isaiah. (1956), “'Equality', Proceedings of the Aristotelian Society". New Series, vol. 56, pp. 281-326. 


\begin{abstract}
Equality in Social Ties: Socio-historical Processes and New Perceptions of Inequality in Chilean Society

The theoretical premise defended by this article is that the contents of equality are specified and transformed according to a society's structural and historical conditioning factors. To argue this hypothesis, the article draws on the results of three studies in Chile, showing that two relevant effects are shaped in the interface between the wave of expansion of the principle of equality in recent decades and society's own characteristics: the rise of new demands for equality, with social ties as their main mechanism of expression, and the deepening of the denouncement of a particular kind of inequalities (defined here as interactional).
\end{abstract}

Key words: equality; social ties; normative principles; social ideals; interactional inequalities; perception of inequalities

\title{
RÉSUMÉ
}

L'Égalité dans le Lien Social: Processus Socio-historiques et Nouvelles Perceptions des Inégalités dans la Société Chilienne

La proposition théorique soutenue dans cet article est que les contenus de l'égalité sont définis et transformés selon les conditionnants structuraux et historiques d'une société. Pour étayer cette hypothèse, on prend appui, dans cet article, sur les résultats de trois recherches empiriques effectuées au Chili, montrant que, dans l'interface entre la vague croissante du principe d'égalité des dernières décennies et les caractéristiques propres à cette société, on remarque deux effets notoires: le surgissement de nouvelles demandes d'égalité, qui contiennent dans le lien social leur principal mécanisme d'expression, ainsi que l'approfondissement de la dénonciation d'un type particulier d'inégalités (qualifiées ici d' interactionnelles).

Mots-clés: égalité; lien social; principes normatifs; système d'idées sociales; inégalités interactionnelles; perception des inégalités. 